\title{
Spatial-Temporal Variability of the Model Characteristics in the Southern Atlantic
}

\author{
I. D. Deinego', I. Ansorge' ${ }^{2}$ K. P. Belyaev ${ }^{1,3, *}$, N. A. Diansky ${ }^{4}$ \\ ${ }^{1}$ Shirshov Institute of Oceanology, Russian Academy of Sciences, Moscow, Russian Federation \\ ${ }^{2}$ University of Cape Town, department of oceanography, Cape Town, South African Republic \\ ${ }^{3}$ Dorodnitsyn Computer Center of Russian Academy of Sciences, Moscow, Russian Federation \\ ${ }^{4}$ Lomonosov Moscow State University, Moscow, Russian Federation \\ *kosbel55@gmail.com
}

Purpose. The present article is aimed at studying spatial-temporal variability of some model characteristics, particularly, the sea level data in the Southern Atlantic.

Methods and Results. The eigenvector decomposition method (called the Karhunen-Loeve decomposition) has been used as a main research technique. Variability of the eigenvectors and eigenvalues of the corresponding covariance matrices, and their distribution in time and space are represented. Application of the method to the problem of assimilating the observation data is shown, and physical sense of such assimilation is analyzed. The ocean hydrodynamics model developed in the Institute of Numerical Mathematics, Russian Academy of Sciences, was applied. The problem of dynamical-stochastic and hybrid assimilation of the sea level data is formulated. Spatial-temporal variability of the model sea level and the one observed in the Southern Atlantic were compared. The variability difference and similarity are analyzed.

Conclusions. The correlation structure between the observed and model ocean level fields is considered. This can permit to assimilate the observational data using the obtained weight matrices. Such studies of the sought characteristics' correlation structures of surface temperature, currents, joint covariance etc. will make it possible to understand exactly how the observed values correct model calculations and to carry out observations in the manner most convenient for data assimilation. Climatic behavior of the structure of eigenvectors and eigenvalues is shown. The represented technique permits to model and to forecast the hydrodynamic processes in the Southern Atlantic in more details.

Keywords: mathematical model, eigenvector and eigenvalue decomposition, dynamical-stochastic data assimilation method, Southern Atlantic.

Acknowledgments: the work is carried out according to the task of the Ministry of Science and High Education of Russian Federation No. 0149-2019-0004, and also at partial support of the RFBR grant No. 19-57-60001. The work of I. Ansorge was supported by the grant UID 118901 of the National Science Foundation of South African Republic.

For citation: Deinego, I.D., Ansorge, I., Belyaev, K.P. and Diansky, N.A., 2019. Spatial-Temporal Variability of the Model Characteristics in the Southern Atlantic. Physical Oceanography, [ejournal] 26(6), pp. 504-514. doi:10.22449/1573-160X-2019-6-504-514

DOI: $10.22449 / 1573-160 \mathrm{X}-2019-6-504-514$

C) 2019, I. D. Deinego, I. Ansorge, K. P. Belyaev, N. A. Diansky

(C) 2019, Physical Oceanography

\section{Introduction}

One of the most important and demanded areas in modern oceanology is the development and application of observational data assimilation (ODA) methods. To give the most complete description of the physical and/or technical dynamic system and its variability at the present stage of research, it is necessary to combine real data of independent observations and calculations by mathematical models and to increase the accuracy of modeling and prediction of physical processes. In recent years, ODA methods have been widely used in the earth 
sciences, providing the relationship between these two main components (observations and models) that allow the environment state monitoring. These methods have been most widely used in meteorology and oceanography, where observations are assimilated into numerical models to obtain boundary or initial conditions, as well as intermediate adjustment of model results for further modeling and forecast [1-3].

ODA methods can be conditionally divided into variational methods based on minimizing a functional given [4-7], which are formulated as optimal control problems and the theory of conjugate equations, and dynamic-stochastic ones, based on probability theory and filtering methods [8-11]. The history of the variational method dates back to the $70 \mathrm{~s}$ of the $20^{\text {th }}$ century, when Guriy. I. Marchuk formulated a fundamental approach to solving the problem of long-term weather forecast. The approach was based on the so-called conjugate equations for nonlinear models of hydrodynamics of the atmosphere and ocean [12, 13]. In dynamic-stochastic ODA schemes, the optimal filtering principle is used, based on the Kalman theory equations [14], now are known as the ensemble Kalman filter methods (EnKF) [15]. Both of the schemes above are actively used in solving problems of operational oceanography, 4D forecast of the ocean state in areas of particular interest from the viewpoint of extraction and transportation of minerals, primarily hydrocarbons, as well as in areas of environmental monitoring, fishing, the location of defence supplies, etc. Such projects as REMO (Brazil) [16], BlueLink (Australia) [17], TOPAZ (Norway) [18] and several others are particularly aimed at the development and application of such ODA methods. There are also a number of hybrid methods that combine both of the approaches above, in addition to basic ODA ones. These methods include, for example, the author ODA scheme [19], in which the functionality constructed using the dynamic-stochastic scheme is minimized.

Nevertheless, in applying both ODA approaches, little attention is paid to the physical understanding of the principles which data assimilation is based on. For example, when applying the dynamic-stochastic ODA method, the following questions: owing to the information is transferred from the observations to the model itself and how exactly the knowledge of the characteristics at some points changes the value of the field in others, - are purely studied or remain unstudied. That is, the relationships between various points of space-time (which are determined by the equations of the model and the corresponding transfer functions), as well as between various model characteristics, are not quantitatively and physically studied. It is not clear which areas are more sensitive to observations and which are not, how such relationships change over time and how quantitatively they depend on the number and density of observations. In the dynamic stochastic approach, these relationships are quantitatively expressed through covariance functions or mutually covariance functions, the construction of which is not an easy task.

In the present work, based on a mathematical model of the general ocean circulation by the Monte Carlo method, covariance matrices of the sea level anomalies are constructed and then these covariance matrices are expanded in eigenvectors. This is a known method in climatology, oceanology, and atmospheric 
physics for decomposition into natural orthogonal components, referred to in the foreign sources as the Karhunen-Loeve decomposition method. The extensive literature is devoted to both the method itself and its application in geophysics. Nevertheless, this method is hardly used for data assimilation, although it makes possible to understand which spatiotemporal relationships of model and the characteristics observed are used for assimilation, and how the spatio-temporal model relationships differ from those observed. In addition, the use of this method can technically simplify the implementation of calculations using the dynamicstochastic approach, since this method allows reducing the size of the covariance matrix. Similar studies were carried out in [20].

In the present paper, the South Atlantic region was chosen for the following reasons. Firstly, this region has been studied much worse than, for example, the North Atlantic one, although its importance for the study of climate and general ocean circulation is not disputed. Secondly, this region is extremely important for countries such as Brazil and South Africa and Russia, as a member of the BRICS, has right and should contribute to the study of processes in the regions of interest to its partners. Finally, the development and application of new methods are more important where there is little data and where their absence or lack can be compensated by the development of more advanced and complex research methods.

\section{Mathematical Model of the General Circulation}

The basic equations of the Institute of Numerical Mathematics Ocean Model (INMOM) general circulation model are defined in a curvilinear orthogonal coordinate system obtained by transforming the Cartesian coordinate system for the generalized sphere and are described by the following system of hydrodynamic equations:

$$
\begin{gathered}
D_{t} u-(l+\xi) v H=\frac{-H}{r_{x}}\left(\frac{1}{\rho_{0}} P_{x}+\frac{1}{\rho_{0}} \frac{\partial p_{a}}{\partial x}-g \frac{\partial \zeta}{\partial x}\right)+\frac{\partial}{\partial \sigma} \frac{n}{H} \frac{\partial u}{\partial \sigma}+\Delta u, \\
D_{t} v-(l+\xi) u H=\frac{-H}{r_{y}}\left(\frac{1}{\rho_{0}} P_{y}+\frac{1}{\rho_{0}} \frac{\partial p_{a}}{\partial y}-g \frac{\partial \zeta}{\partial y}\right)+\frac{\partial}{\partial \sigma} \frac{n}{H} \frac{\partial v}{\partial \sigma}+\Delta v, \\
\operatorname{div}_{h} u+\frac{1}{H} \frac{\partial w}{\partial \sigma}=\frac{1}{H} \frac{\partial \zeta}{\partial t}, \\
\tilde{D_{t}} \theta=\frac{\partial}{\partial \sigma} \frac{n_{\theta}}{H} \frac{\partial \theta}{\partial \sigma}+\Delta \theta+\frac{\partial R}{\partial \sigma}, \\
\tilde{D_{t} S}=\frac{\partial}{\partial \sigma} \frac{n_{S}}{H} \frac{\partial S}{\partial \sigma}+\Delta S, \\
\rho=\hat{\rho}\left(\theta, S+35, p_{w}\right)-\hat{\rho}\left(0,0, \rho_{0} g \sigma H\right) .
\end{gathered}
$$

* Rozhkov, V.A., 2015. [Statistical Hydrometeorology. Part 3: State and Movement Instability. The Ocean and Amosphere Interaction. Climate]. St. Petersburg: Izd-vo SpbGU. 255 p. (in Russian). 
Here $u=(u, v)$ is the horizontal velocity vector, $u$ and $v$ are zonal and meridional components of the current velocity; $w$ is the vertical velocity in the $\sigma$-coordinate system, where $\sigma=(z-\zeta(x, y, t)) /(H(x, y)-\zeta(x, y, t)), z$ is the downward vertical coordinate, $\zeta$ is the sea level deviation from an unperturbed surface, $H$ is the ocean depth at rest; $\theta$ is the potential temperature; $R$ is the penetrating solar radiation flux; $S$ - salinity excluding a constant equal to 35PSU; $\rho$ is the water density deviation from some average density profile, depending only on the pressure of the liquid column $\rho_{0}$ with an average density in the ocean $\rho_{0}=1.025 \mathrm{~g} / \mathrm{cm}^{3}$ at the depth $z=\sigma H ; \quad l$ is the Coriolis parameter; $\Delta$ is the horizontal gradient operator. The metric coefficients $r_{x}$ and $r_{y}$ are set in advance when choosing a generalized coordinate system, and the viscosity and diffusion coefficients $n, n_{\mathrm{s}}$ and $n_{\theta}$ are considered known. The $D_{t}$ operator is the so-called transport operator, which, when applied to an arbitrary scalar variable $\varphi$ (for example, temperature or salinity), is written as follows

$$
\tilde{D}_{t} \varphi=\frac{\partial \xi \varphi}{\partial t}+\left(r_{x} r_{y}\right)^{-1}\left[\frac{\partial}{\partial x}\left(r_{y} u \varphi\right)+\frac{\partial}{\partial y}\left(r_{x} v \varphi\right)\right]+\frac{\partial}{\partial \sigma} w \varphi .
$$

Other designations are commonly understood.

This model is described in detail in [21, 22]; the configuration of the model and its experimental implementation are important in the present study. The model is implemented for the joint water area of the Atlantic and the Arctic basin, including the Bering Sea, from Antarctica to the Aleutian Islands with a spatial resolution of $0.25^{\circ}$ in the coordinate system rotated $90^{\circ}$ so that the model poles lie at the geographic equator. Forty unevenly located $\sigma$-levels are set in depth with a more frequent definition in the upper layers.

For solid sections of the lateral boundary, the non-flow and free slip conditions are used for the velocity of the currents, and at the bottom, the non-flow and quadratic bottom friction conditions re used. For temperature and salinity in all areas of the solid boundary, the isolation conditions are set.

On the aquatic boundaries in the Drake Strait, between Africa and Antarctica, and between the Bering Sea and the Pacific Ocean, the average long-term monthly values of temperature and salinity from the atlas [23] were prescribed. Since density values were set at aquatic boundaries (through temperature and salinity), the condition for setting geostrophic current velocities is automatically realized here. As the river runoff, the monthly climate average water consumption data for all large rivers of the model water area were used, and to set the atmospheric effect (external forcing) - the climatic surface data from the NCEP/NCAR atlas [24], as proposed in the Common Ocean Research Experiment (CORE) project.

Equations (1) - (6) are integrated from zero initial conditions for velocities and values from the atlas [23] for temperature and salinity for 40 years (the so-called spin-up procedure). As a result of the spin up, the obtained fields for the last 8 years are recorded for each day. Thus, there are $365 \times 8$ daily climatic values (leap years are not taken into account). The velocity of the currents and the level 
field are constructed from these fields. All calculations were carried out on the Lomonosov-1 Supercomputer of Lomonosov Moscow State [25].

Fig. 1 shows the surface current velocities calculated according to model (1) (6) for summer (January 15, an average of 8 years) and winter (July 15, an average of 8 years). As seen, the seasonal climatic currents slightly differ, although there are certain differences: in winter the currents intensify, especially in the Drake Strait, the velocities are slightly higher and the horizontal gradient increases.
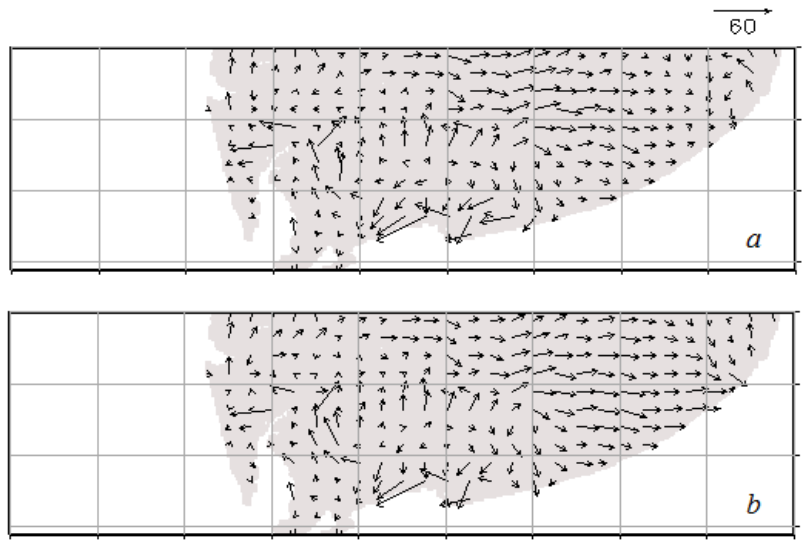

F i g. 1. Model fields of surface currents in the Drake Strait region in summer $-a$ and in winter $-b$. Model coordinate system. The left aquatic boundary is in the Drake Strait, the right one - between Antarctica and Africa

In Fig. 2 shows the similarly constructed fields of level $\zeta$. It is also noticeable that in winter the maximum level zone is somewhat shifted to the north, in the summer a small additional zone of high values of the level appears near Antarctica, the gradient is blurred.

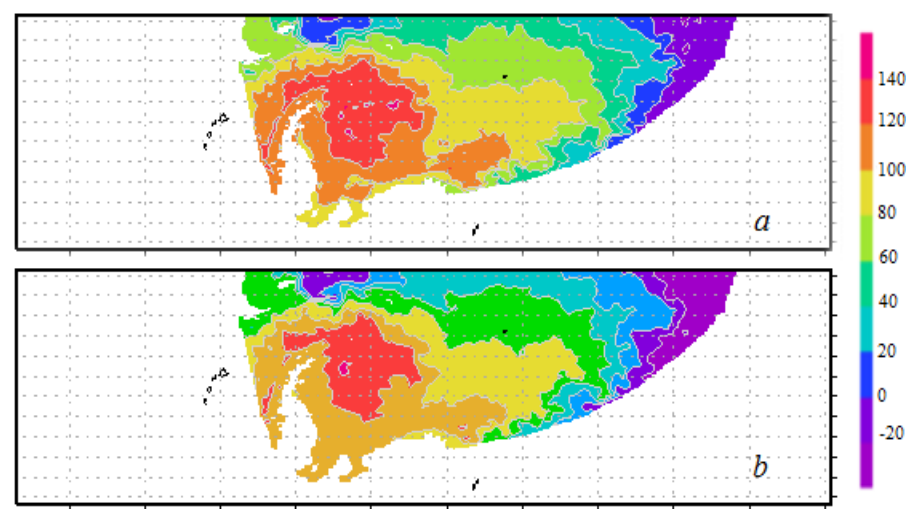

F i g. 2. Model fields of the ocean level in the Drake Strait region in summer $-a$ and in winter $-b$. Model coordinate system. The left aquatic boundary is in the Drake Strait, the right one - between Antarctica and Africa. Positive values correspond to the level lowering 
Model level values can be compared with the observed level according to Altimetry Validating and Interpolating Satellite Observations (AVISO) (www.aviso.gov). Fig. 3 shows the ocean level for climate January. Compared to the model level (Fig. 2, a) in Fig. 3, synoptic eddies in the Circumpolar Current region are more pronounced, and large-scale structures in the western part of the South Atlantic are less noticeable. However, the overall picture of the level is similar both quantitatively and qualitatively.

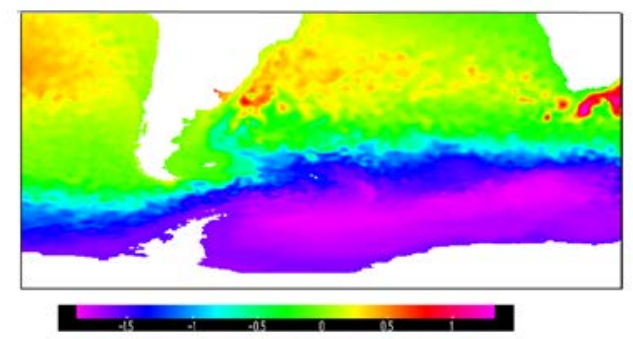

F i g. 3. Observed sea surface level (m) in the Drake Strait region in January. AVISO data

\section{Covariance relations in the observational data assimilation}

During the ODA according to the dynamic stochastic scheme, the following algorithm is used:

$$
\begin{aligned}
& X_{a}=X_{b}+K\left(Y-H X_{b}\right), \\
& K=B H^{T}\left(H B H^{T}+R\right)^{-1} .
\end{aligned}
$$

Here $X_{a}$ and $X_{b}$ are the model fields (conditions of the model) after (analysis) and before (background) correction, correspondingly; $Y$ is the vector of observations; $K$ is the weight matrix (Kalman gain); $B$ is the so-called covariance matrix of the model ensemble. It is rather difficult to give a strict definition of it; instead, the method of its construction is usually described, namely: the $X^{l}$ ensemble is constructed, where $l=1, \ldots, N$, of the model calculations with different initial conditions and then at each point of the grid $i, j$ a matrix of the type is constructed

$$
B=\left\{b_{i j}\right\}=N^{-1} \sum_{l=1}^{N}\left(X_{i}^{l}-\overline{X_{i}}\right)\left(X_{j}^{l}-\overline{X_{j}}\right)^{T},
$$

where $\overline{X_{i}}$ is the ensemble mean in $i$ point. In addition, projection operator $H$ is included in (8) and (9) (the basic concept of which is to project the model value at the observation point and at the same time to remove all unobserved variables from consideration), as well as the diagonal matrix $R$ (the physical meaning of which is the variance of instrumental observation errors ), which is usually given empirically. The superscript $T$ denotes the transposition of the vector and/or matrix.

As seen from formula (10), the B matrix is quite awkward, it depends on each pair of grid points (as well as on the size of the ensemble) and its construction and analysis is a difficult task. Nevertheless, it is from the B matrix that the K matrix weight is determined, which represents the transfer function of the observations, or rather, the difference between the observation and the model, i.e., from the calculation error to the model itself. 
In the literature, the $\mathrm{B}$ matrix construction and then the $\mathrm{K}$ matrix weight is the main task of ODA according to the dynamic stochastic scheme. There are very few works in which the properties of these matrices are studied, as well as any comparisons of model matrices with those constructed from observations are made. In addition to the aforementioned work [20], we also call [26], where the numerical characteristics of the B matrix are studied. Meanwhile, as can be seen from formulas (8) and (9), the structure and spectrum of the symmetric $H B H^{T}$ matrix will determine the $\mathrm{K}$ matrix properties and then $X_{a}$ analysis. With successive assimilation, the temporal characteristics of these matrices will make the main contribution to the temporal variability of the corrected field. Moreover, the physical properties of the field obtained, for example, the characteristics of waves or $f$, directly depend on how well these matrices take into account the physical relationships of the model and observed parameters. in view of the aforesaid, before directly assimilating the data using formulas (8) and (9) we made an attempt to deal with these relationships.

The results of the last 8 years of model calculations with climatic forcing for every day were presented in the previous section. Based on these data, 8-year averages and anomalies (the difference between the current and mean values) were constructed. Then, covariance matrices of anomalies were constructed using the formula (10). At the same time, for each climatic month, the $15 \mathrm{t}^{\mathrm{h}}$ day (the middle day of the month) was selected and to increase the sample length, the $5^{\text {th }}, 10^{\text {th }}, 20^{\text {th }}$ and $25^{\text {th }}$ dates of the same month were added, i.e., the sample length was $5 \times 8=40$. Totally, 12 monthly average covariance matrices of anomalies with a sample length of 40 were constructed.

\section{Analysis of the results and some conclusions}

For the study, the ocean level indicated above as $\zeta$ was chosen as the main physical characteristics. According to the formula (10), the covariance functions of anomalies of this level for each climatic month were constructed. Since only model data was considered, the H operator in the formula (10) was chosen to be unit.

For the $H B H^{T}$ matrix, which in what follows will be denoted by $B$, eigenvectors and values were looked for. That is, such a set of vectors $X^{I}$ was searched, where $l=1, \ldots, L\left(L-2 \mathrm{D}\right.$ grid dimension), at which $B X^{m}=\lambda_{m} X$, where $\lambda_{m}$ are eigenvalues. In the present case the dimension was $L=148 \times 764$. Then the representation of any vector, including the difference between the observed and model fields $\left(Y-H X_{b}\right)$, can be expressed as follows

$$
X_{a}=X_{b}+K\left(Y-H X_{b}\right)=X_{b}+\sum_{m=1}^{L} \alpha_{m} \lambda_{m} X^{m},
$$

where $\alpha_{m}$ are some coefficients determined by the observed vector decomposition. Structure of the analysis fields will be completely determined by the characteristics of the eigenvectors $X^{m}$, and their total contribution by the values of the eigenvalues $\lambda_{m}$, taking into account the fact that the eigenvectors of the direct and inverse symmetric matrices coincide, and the eigenvalues of the inverse matrix are the contrary (i.e., they are raised to the first power minus) to the eigenvalues of a direct matrix. 


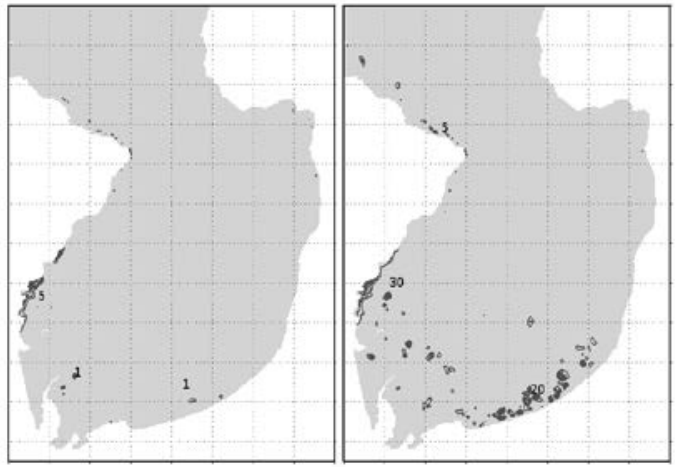

F i g. 4. The first (left) and the second (right) eigenvectors in summer

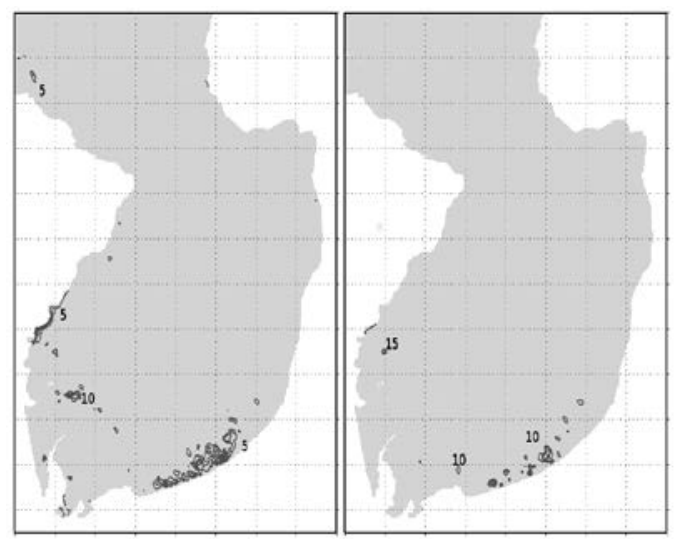

F i g. 5. The first (left) and the second (right) eigenvectors in winter

Fig. 4 shows the first two eigenvectors of the $\zeta^{m}$ level anomaly, where $m=1,2$ for January (summer). Noteworthy is the presence of noticeable nonzero values along the coast, especially in the southern part of the ocean, and the almost complete absence of any signal in the region center. This means that the main covariance relations, i.e., the correlation between the spatial values of the level, are transmitted precisely along these nonzero values. It is easy enough to identify these connections with known wave structures, in particular with shelf trapped waves, which are very similar in structure.

Winter (July) covariance relationships are shown in Fig. 5. Here, intensification of values along the southern coast, as well as a strong stream along the South American shelf, which are not traced in the summer, are noticeable. In general, both eigenvectors in both winter and summer show the concentration of interconnections along the southern Atlantic, closer to the African and Antarctic coast, and to a lesser extent along South America.

The structure of the eigenvalues corresponding to the vectors constructed earlier should be also presented. Fig. 6 shows the normalized values of the first two components in the annual course. It can be seen that the first component is approximately three times greater in value and, consequently, in energy, than 
the second component of the corresponding matrix. These eigenvalues have a pronounced climatic seasonal variation; they noticeably decrease in value in winter (May - September) and increase towards summer (November - February). Moreover, the structure of eigenvectors itself also has a seasonal variation, but manifests itself in the opposite way: in January it is much poorer than in July.

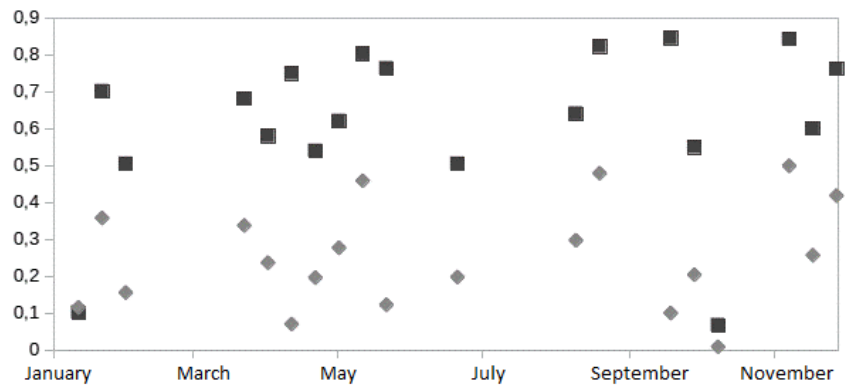

F i g. 6. Distribution of the eigenvalues of two main components normalized for the sum: $\mathbf{a}-$ the first component, $\$$ - the second one

\section{Conclusion}

The problem of numerical modeling of the South Atlantic hydrodynamics with the relationship structure analysis of the characteristics of the observed and model level fields is considered. These calculations will make it possible to further assimilate the observational data and understand how exactly this data affect the model fields and the characteristics associated. The study of such structures and relationships allows not only to understand how the observed values correct the model calculation, but also to identify particularly important areas for assimilation; to understand where it is necessary to carry out observations so that the observed information is used in the most effective way.

The paper shows that the structure of eigenvectors and numbers has a noticeable climatic seasonal variation. Eigenvectors have a richer and more energetically pronounced structure in winter (July) compared to summer (January). On the contrary, the eigenvalues corresponding to these vectors behave in the opposite way - they are maximal in summer and minimal in winter.

The expansion of the covariance functions applied for ODA can significantly improve the computational characteristics of the assimilation algorithm, speed up the procedures for finding weight transfer matrices and generally simplify the ODA scheme itself. In addition, it is possible to compare preliminarily the observed and model structures and their relationships to determine the most energetically important structures, to study their seasonal and synoptic variability.

In the present article, as a first step, all necessary calculations were carried out for level fields. However, nothing prevents to do the same for fields of ocean surface temperature, surface currents, as well as for determining joint covariance relations.

In the future, it is planned to assimilate observational data from hydrological stations carried out in expeditions together with the University of Cape Town, South Africa. The application of this technique, as well as other modern methods and models, will make it possible to understand the hydrodynamic processes in the South Atlantic in more detail. 


\section{REFERENCES}

1. Ghil, M. and Malanotte-Rizzoli, P., 1991. Data Assimilation in Meteorology and Oceanography. Advances in Geophysics, [e-journal] 33, pp. 141-266. doi:10.1016/S0065-2687(08)60442-2

2. Kalnay, E., Ota, Y., Miyishi, T. and Liu, J., 2012. A Simpler Formulation of Forecast Sensitivity to Observations: Application to Ensemble Kalman Filters. Tellus A: Dynamic Meteorology and Oceanography, [e-journal] 64(1), 18462. https://doi.org/10.3402/tellusa.v64i0.18462

3. Marchuk, G.I., Paton, B.E., Korotaev, G.K. and Zalesny, V.B., 2013. DataComputing Technologies: A New Stage in the Development of Operational Oceanography. Izvestiya, Atmospheric and Oceanic Physics, [e-journal] 49(6), pp. 579-591. https://doi.org/10.1134/S000143381306011X

4. Marchuk, G.I., and Zalesny, V.B., 2012. Modeling of the World Ocean Circulation with the Four-Dimensional Assimilation of Temperature and Salinity Fields. Izvestiya, Atmospheric and Oceanic Physics, [e-journal] 48(1), pp. 15-29. https://doi.org/10.1134/S0001433812010070

5. Agoshkov, V.I., Ipatova, V.M., Zalesnyi, V.B., Parmuzin, E.I. and Shutyaev, V.P., 2010. Problems of Variational Assimilation of Observational Data for Ocean General Circulation Models and Methods for their Solution. Izvestiya, Atmospheric and Oceanic Physics, [e-journal] 46(6), pp. 677-712. doi:10.1134/S0001433810060034

6. Agoshkov, V.I., Parmuzin, E.I. and Shutyaev, V.P., 2008. Numerical Algorithm for Variational Assimilation of Sea Surface Temperature Data. Computational Mathematics and Mathematical Physics, [e-journal] 48(8), pp. 1293-1312. doi:10.1134/S0965542508080046

7. Marchuk, G.I. and Zalesny, V.B., 1993. A Numerical Technique for Geophysical Data Assimilation Problem Using Pontryagin's Principle and Splitting-up Method. Russian Journal of Numerical Analysis and Mathematical Modelling, [e-journal] 8(4), pp. 311-326. https://doi.org/10.1515/rnam.1993.8.4.311

8. Xie, J. and Zhu, J., 2010. Ensemble Optimal Interpolation Schemes for Assimilating Argo Profiles into a Hybrid Coordinate Ocean Model. Ocean Modelling, [e-journal] 33( 3-4), pp. 283-298. doi:10.1016/j.ocemod.2010.03.002

9. Korotaev, G.K., Knysh, V.V., Lishaev, P.N. and Demyshev, S.G., 2018. Application of the Adaptive Statistics Method for Reanalysis of the Black Sea Fields Including Assimilation of the Temperature and Salinity Pseudo-measurements in the Model. Physical Oceanography, [e-journal] 25(1), pp. 36-51. doi:10.22449/1573-160X-2018-1-36-51

10. Belyaev, K.P., Tanajura, C.A.S. and Tuchkova, N.P., 2012. Comparison of Methods for Argo Drifters Data Assimilation into a Hydrodynamical Model of the Ocean. Oceanology, [e-journal] 52(5), pp. 593-603. doi:10.1134/S0001437012050025

11. Belyaev, K., Kuleshov, A. Tuchkova, N. and Tanajura, C.A.S., 2017. An Optimal Data Assimilation Method and Its Application to the Numerical Simulation of the Ocean Dynamics. Mathematical and Computer Modelling of Dynamical Systems, [e-journal] 24(1), pp. 12-25. doi:10.1080/13873954.2017.1338300

12. Marchuk, G.I., 1970. Numerical Methods of Weather Forecasting. Wright-Patterson Air-Force Base, Ohio: Foreign Technology Division, $391 \mathrm{p}$.

13. Marchuk, G.I. and Penenko, V.V., 1979. Application of Optimization Methods to the Problem of Mathematical Simulation of Atmospheric Processes and Environment. In: G. I. Marchuk, ed., 1979. Modelling and Optimization of Complex Systems. Lecture Notes in Control and Information Sciences, vol. 18. Berlin, Heidelberg: Springer, pp. 240-252. doi:10.1007/BFb0004167

14. Kalman, R.E., 1960. A New Approach to Linear Filtering and Prediction Problems. Journal of Fluids Engineering, [e-journal] 82(1), pp. 35-45. doi:10.1115/1.3662552

15. Evensen, G., 2009. Data Assimilation: The Ensemble Kalman Filter. Berlin: Springer, 307 p. https://doi.org/10.1007/978-3-642-03711-5

16. Lima, M.O., Cirano, M., Mata, M.M., Goes, M., Goni, G. and Baringer, M., 2016. An Assessment of the Brazil Current Baroclinic Structure and Variability near $22^{\circ} \mathrm{S}$

PHYSICAL OCEANOGRAPHY VOL. 26 ISS. 6 (2019) 
in Distinct Ocean Forecasting and Analysis Systems. Ocean Dynamics, [e-journal] 66(6-7), pp. 893-916. doi:10.1007/s10236-016-0959-6

17. Schiller, A. and Brassington, G.B., 2011. Operational Oceanography in the 21st Century. Dordrecht: Springer, 745 p. https://doi.org/10.1007/978-94-007-0332-2

18. Cummings, J.A. and Smedstad, O.M., 2013. Variational Data Assimilation for the Global Ocean. In: S. Park, L. Xu, eds., 2013. Data Assimilation for Atmospheric, Oceanic and Hydrologic Applications (Vol. II). Berlin, Heidelberg: Springer, pp. 303-343. https://doi.org/10.1007/978-3-642-35088-7_13

19. Belyaev, K.P., Kuleshov, A., Smirnov, I. and Tanajura, C.A.S., 2019. A Hybrid Data Assimilation Method and Its Comparison with an Ensemble Optimal Interpolation Scheme in Conjunction with the Numerical Ocean Model Using Altimetry Data. Ocean Science Discussion, [e-journal] https://doi.org/10.5194/os-2019-56

20. Belyaev, K.P. and Tanajura, C.A.S., 2013. A Time-Space Description of the Analysis Produced by a Data Assimilation Method. In: R. Rubio [et al.] (eds.), 2013. Without Bounds: A Scientific Canvas of Nonlinearity and Complex Dynamics. Berlin: Springer, pp. 729-746. https://doi.org/10.1007/978-3-642-34070-3_52

21. Frey, D.I., Morozov, E.G., Fomin, V.V. and Diansky, N.A., 2018. Spatial Structure of the Antarctic Water Flow in the Vema Fracture Zone of the Mid-Atlantic Ridge. Izvestiya, Atmospheric and Oceanic Physics, [e-journal] 54(6), pp. 621-625. https://doi.org/10.1134/S0001433818060063

22. Gusev, A.V. and Diansky, N.A., 2014. Numerical simulation of the world ocean circulation and its climatic variability for 1948-2007 using the INMOM. Izvestiya, Atmospheric and Oceanic Physics, [e-journal] 50(1), pp. 1-12. https://doi.org/10.1134/S0001433813060078

23. Levitus, S.E., 1982. Climatological Atlas of the World Ocean. Princeton: NOAA, 173 p.

24. Kalnay, E., 2002. Atmospheric Modeling, Data Assimilation and Predictability. New York: Cambridge University Press, 341 p. https://doi.org/10.1017/CBO9780511802270

25. VVoevodin, V1., Jumatii, S., Sobolev, S., Antonov, A., Bryzgalov, P., Nikitenko, D., Stefanov, K. and Voevodin, Vad., 2012. [The Practice of the Lomonosov Supercomputer]. Open Systems. DBMS, (7), pp. 36-39 (in Russian).

26. Kaurkin, M.N., Ibraev, R.A. and Belyaev, K.P., 2018. Assimilation of the AVISO Altimetry Data into the Ocean Dynamics Model with a High Spatial Resolution Using Ensemble Optimal Interpolation (EnOI). Izvestiya, Atmospheric and Oceanic Physics, [e-journal] 54(1), pp. 56-64. https://doi.org/10.1134/S0001433818010073

About authors:

Irina D. Deinego $-2^{\text {nd }}$ year postgraduate, P.P. Shirshov Institute of Oceanology (36 Nahimovskiy pr., Moscow, 117997, Russian Federation), ORCID ID: 0000-0003-2985-028X, deynego.id@ocean.ru

Isabelle Ansorge - Professor, University of Cape Town Oceanography Department (Rondebosch, 7701, RSA), Ph. D., ORCID ID: 0000-0001-7071-8147, isabelle.ansorge@uct.ac.za

Konstantin P. Belyaev - Senior Research Scientist, Shirshov Institute of Oceanology, RAS; Federal Research Center of Control, Dorodnitsyn Computer Center RAS (40 Vavilov St., 119333, Moscow, Russian Federation), Dr.Sci (Phys.-Math.), Scopus Author ID: 6603447653, kosbel55@gmail.com

Nikolay A. Diansky - Leading Research Associate, Lomonosov Moscow State University (GSP-1, Leninskie Gory, Moscow, 119991, Russian Federation), Dr.Sci (Phys.-Math.), Associate professor, ORCID ID: 0000-0002-6785-1956, ResearcherID: R-8307-2018, nikolay.diansky@gmail.com

Contribution of the co-authors:

Irina D. Deinego - the main author, carried out the calculations

Isabelle Ansorge - contributed to the description of regional features in the discussion of the results

Konstantin P. Belyaev - suggested a method and took part in writing an article

Nikolay A. Diansky - the author of the model and contributed to the writing of the article

All the authors have read and approved the final manuscript.

The authors declare that they have no conflict of interest. 